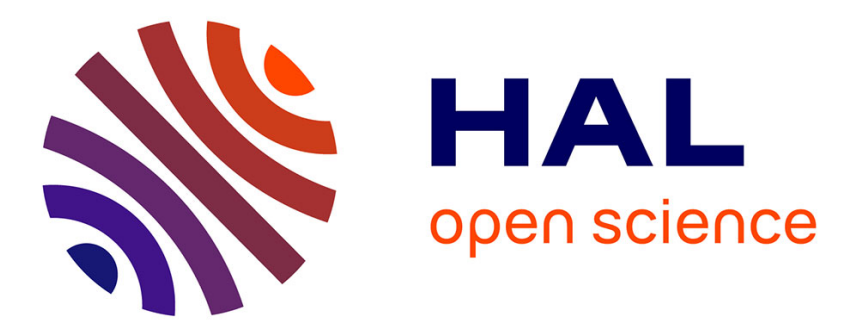

\title{
On-site airflow measurement of a laboratory fume hood using customized large-scale image-based velocimetry
}

Romain Schuster, Dominique Heitz, Philippe Georgeault, Etienne Mémin

\section{To cite this version:}

Romain Schuster, Dominique Heitz, Philippe Georgeault, Etienne Mémin. On-site airflow measurement of a laboratory fume hood using customized large-scale image-based velocimetry. Indoor and Built Environment, 2020, 29 (6), pp.810-819. 10.1177/1420326X19865928 . hal-02419135

\section{HAL Id: hal-02419135 \\ https://hal.science/hal-02419135}

Submitted on 19 Dec 2019

HAL is a multi-disciplinary open access archive for the deposit and dissemination of scientific research documents, whether they are published or not. The documents may come from teaching and research institutions in France or abroad, or from public or private research centers.
L'archive ouverte pluridisciplinaire HAL, est destinée au dépôt et à la diffusion de documents scientifiques de niveau recherche, publiés ou non, émanant des établissements d'enseignement et de recherche français ou étrangers, des laboratoires publics ou privés. 


\title{
On-site airflow measurement of a laboratory fume hood using customized large-scale image-based velocimetry
}

\section{Romain Schuster ${ }^{1,2,3}$, Dominique Heitz ${ }^{2,3}$, Philippe Georgeault ${ }^{2}$ and Etienne Mémin ${ }^{3}$}

\begin{abstract}
This study demonstrates the feasibility of conducting on-site large-scale image-based measurements for indoor airflows characterisation. To illustrate the potential of our method, we chose to study the suction flow generated by a laboratory fume hood in operating conditions. As a matter of fact laboratory fume hoods are frequently subject to routine checks during which air speed measurements by hot-wire anemometry are performed. However classical point-to-point hot-wire anemometry may not be sufficient to detect and localize potential leakages. To improve these controls we developed and tested a new method based on particle image velocimetry principles, which is non-intrusive and authorizes a good spatio-temporal analysis. To face large-scale and on-site issues we had to make some adaptations. For this reason, we used tracers like bubbles or smoke which have good scattering properties. We also developed our own low-cost light system. To compute velocities from image sequences we developed an optical flow algorithm based on a large-scale flow model instead of using traditional correlation. The tested method gave good results with a good agreement with sparse HWA measurements but with a wider spatial distribution. In addition the method provided turbulence intensity estimation and a good monitoring of dynamic flow features, which is important for leakages detection.
\end{abstract}

\section{Keywords}

Laboratory fume hood, Large-scale velocimetry, Optical flow, Air leakage detection, Turbulence intensity

\section{Introduction}

Laboratory fume hoods are protection devices generally used in places where harmful substances are handled. They are aimed at limiting the exposure of humans to contaminants by capturing and removing hazardous fumes or dust generated inside the enclosure thanks to a suction flow. To insure their good performances through time, fume hoods are subject to periodical controls. These controls usually consist of a three-part evaluation process composed by a face flow rate evaluation, a qualitative smoke visualisation and a tracer gas test. This protocol does not give any information about the presence or the location of potential leakages. To detect potential pollutants leakages, it is necessary to understand the aerodynamics features of the fume hood suction flow. Studies like [Tseng et al. (2006)] and [Chen (2016)] tried to understand the effect of the perturbations induced by the presence of a manikin on the flow pattern. They also investigated the impact of the sash handle shape on the containment. However in these studies the airflow pattern was brought to light thanks to qualitative smoke visualisation solely, no quantitative velocity measurements were performed. Actually, the possibility of experimentally extracting dense quantitative flow motion estimation from smoke visualisation images has been proved [Heitz et al. (2010)]. This is one of the things we propose to apply in this paper. More recently, the two studies [Liu et al. (2017)] and [Pietrowicz et al. (2018)] numerically investigated the fume hood airflow with quantitative results. It would be interesting to compare these results to an experimental database.

In this study we propose to perform an on-site, largescale, image-based air velocity measurement on a laboratory fume hood in operating conditions. Traditionally particle image velocimetry (PIV) technique is carried out to spatially estimate fluid motion. Briefly this method consists of seeding a flow with tracer particles and illmminate

\footnotetext{
1 ITGA, Saint-Grégoire Cedex 35768, France

2 Irstea, UR OPAALE, Rennes Cedex F-35044, France

${ }^{3}$ Inria, Fluminance group, 35042 Rennes, France
}

Corresponding author:

Romain Schuster, ITGA, Saint-Grégoire Cedex 35768, France

Email: romain.schuster@itga.fr 
illuminating them in a $2 \mathrm{D}$ slice thanks to a laser sheet. Simultaneously particle images are recorded and particle displacements between successive frames are computed giving thus an estimation of the flow velocity. This method, originally introduced by [Adrian (1991)], is now widely used in the fluid mechanics community to study turbulent flows. PIV principles and advances have been carefully explained and detailed in the books Particle Image Velocimetry [Raffel et al. (2018)] and [Adrian and Westerweel (2011)]. Image-based velocimetry techniques like PIV are non-intrusive and provide instantaneous velocity fields. PIV was originally designed for laboratories and well-controlled flows, and its transposition to an on-site measurement tool is not immediate. Some ventilation or air conditioning systems inside cars, planes or rooms were investigated using PIV as mentioned in the review [Cao et al. (2014)] but experiments were mostly carried out in research laboratories on specifically designed models and rarely on-site in real conditions. The Airflow generated by a range hood (which is not exactly a laboratory fume hood) has been quantitatively measured thanks to $2 \mathrm{D}$ PIV [Jiang et al. (2016); Chen (2018)]. However in the latter one, measurements has been performed on a specifically designed mockup of a kitchen cabinet built inside a laboratory. They used a camera with high resolution. Thus performing PIV on-site and in real conditions needs some adaptations. As fields of investigation are getting bigger, classical particles like oil droplets cannot be used as tracer because they are too small to backscatter enough light and to be seen by the camera. Two options are then possible: using bigger particles like Helium Filled Soap Bubbles (HFSB) or using dense smoke seeding. The advantage of HFSB is that they are big enough (diameter around $300 \mu \mathrm{m}$ ) to strongly scatter the light and being visible from far. Secondly thanks to the helium, bubbles have the neutral buoyancy needed to follow the flow with high fidelity [Scarano et al. (2015)]. The interest of employing a smoke generator is it simplicity and availability. Smoke is compound of oil droplet haze scattering the light due to the high density but giving rise to smooth scalar images. In scalar images we track the luminance gradients motion. This type of images are difficult to analyse with correlation technique, which is the standard processing to compute displacements fields from particle image sequences. In the other hand optical flow approaches [Heitz et al. (2010)] are actually better to estimate fluid motion from scalar images. This is why we prefered to develop our own optical flow algorithm additionally taking into account that with such images we estimate the largest scales of the flow velocity [Schuster et al.
(2019)]. The following study is thus aimed at demonstrating the feasibility of an on-site PIV-like measurement of a fume hood airflow in operating conditions by using suited tracers and a dedicated algorithm. We will compare the two kinds of tracers mentioned above: HFSB and smoke, in order to determine what tracer is the best suited for large-scale onsite flow characterisation. The rest of this paper is organised as follows. In Section 2, the image-based method is described in detail: we present the material and the motion estimation method. Then results and performances of the method are presented and commented in Section 3. Finally, conclusion and perspectives are discussed in Section 4.

\section{Material and method}

The method consists of bringing to light the invisible airflow generated by a laboratory fume hood. To make it possible, the flow is seeded with a tracer (particles or smoke). In the same time a light sheet is emitted by an illumination system in order to enlighten the tracers in the zone of interest. The light scattered by the tracer is recorded by a camera at a given frame rate. By computing tracer's displacements between successive frames thanks to a motion estimation method it is possible to obtain an estimation of the velocity field in the illuminated slice. Naturally as we only illuminate a crosssection and use only one camera we can only retrieve two components of the velocity in two directions (2D2C).

\subsection{Experimental setup}

Usually, to perform a PIV measurement a Nd:YAG pulsed laser at $532 \mathrm{~nm}$ combined with a cylindrical lens is employed to generate a light sheet. The light delivered by a laser is powerful enough for large-scale applications but the whole light system is not easy to move everywhere and is very expensive. Moreover for ocular safety questions laser is not recommended for on-site diagnosis. Therefore we have chosen to develop, in collaboration with the french company EFFILUX $^{1}$, our own lighting system more transportable, easy to handle and less expensive. Our device consists of an array of LEDs coupled with a linear Fresnel lens that produces a collimated blue light sheet. LEDs were chosen to emit at $462 \mathrm{~nm}$ because of their better efficiency at this wavelength. The LEDs array generates a $1 \mathrm{~m}$ high and about $10 \mathrm{~mm}$ thick light sheet. This lighting system can be run both in trigger mode or in continuous mode. The advantage of trigger mode is the possibility of scaling up the light power for a short duration. In our configuration the LEDs array was placed vertically facing the fume hood front opening. Image recordings were acquired with a CCD camera Photron 


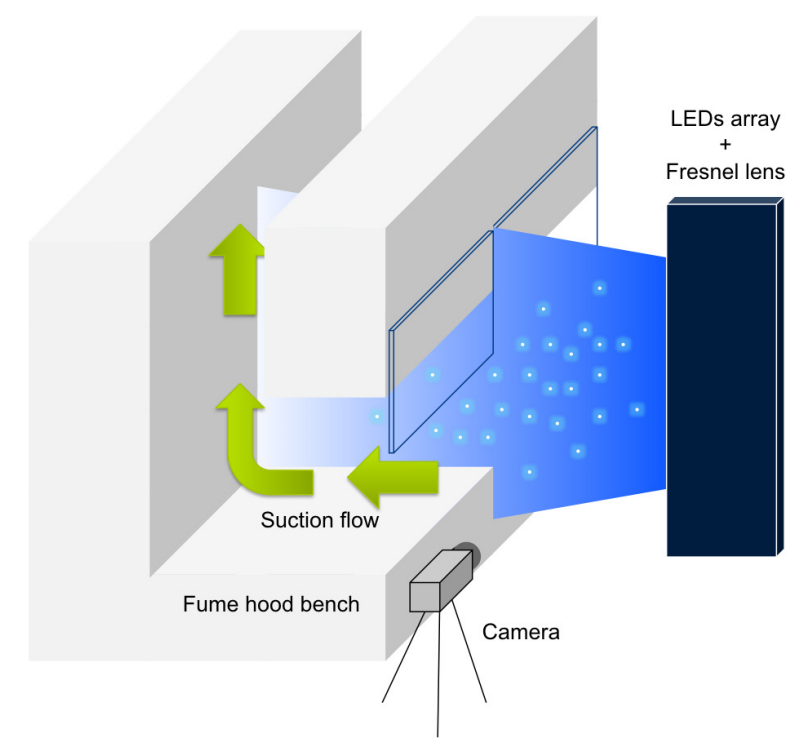

Figure 1. Sketch of the experimental setup.

equipped with a $35 \mathrm{~mm}$ focal lens and storing $1024 \times$ 1024 pixel $^{2} 8$ bits images at 60,125 or 250 fps. The results presented in this paper were acquired with a continuous light to maximise the amount of light received by the CCD sensor. As the studied flow was quite slow the blur effect due to continuous light was very soft. The camera was positioned perpendicular to the illuminated plane at a distance of $1.5 \mathrm{~m}$ from it. As we were constrained by the geometry of the room, the field of view was limited and the interior of the fume hood was truncated in the longitudinal direction (see figure 2). About the seeding systems, we used the HFSB generator developed by LaVision ${ }^{2}$. The HFSB generator that we used produces small bubbles down to $300 \mu \mathrm{m}$ diameter size with a high seeding density (up to 2 million bubbles per second). For the smoke we used a basic theatrical fogger. The laboratory fume hood used for this study was located at the Irstea center of Rennes. Experiments were conducted for a sash window height of $500 \mathrm{~mm}$. For this study the fume hood was unoccupied. We think that performing our method with a manikin should be relevant to see the influence of human body on the flow, but this will be carried out in further studies. A global overview of the experimental setup is illustrated in figure 1. As it can be seen in figure 2(a) the room was in darkness but no black background was needed with the HFSB seeding since the bubbles scatter the light very strongly. In contrary, in figure 2(b) a black background had to be added to make the smoke more visible. One of the main difficulty was to introduce the tracer in the light sheet axis.

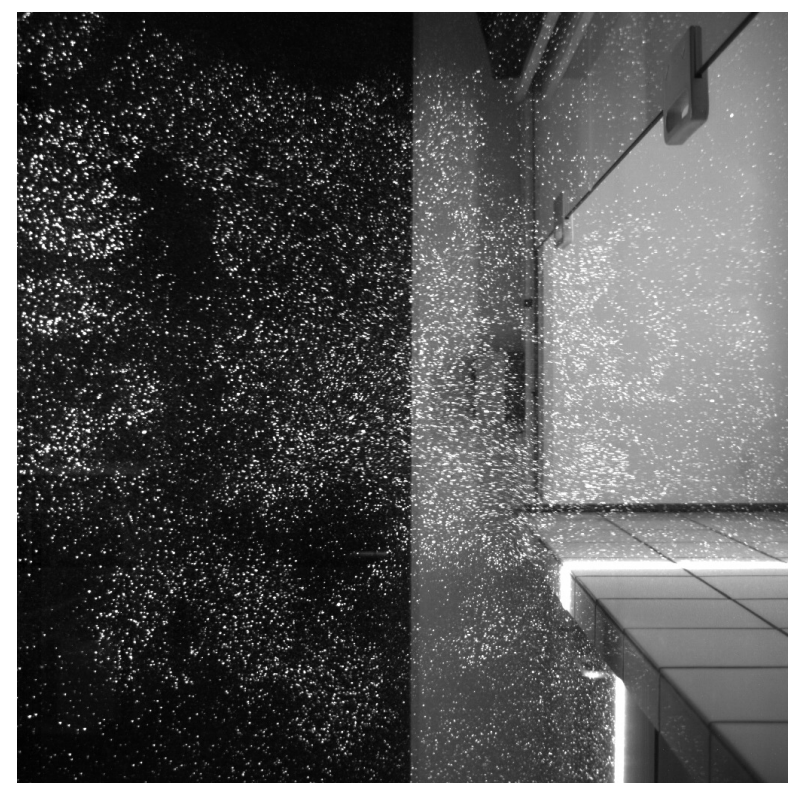

(a)

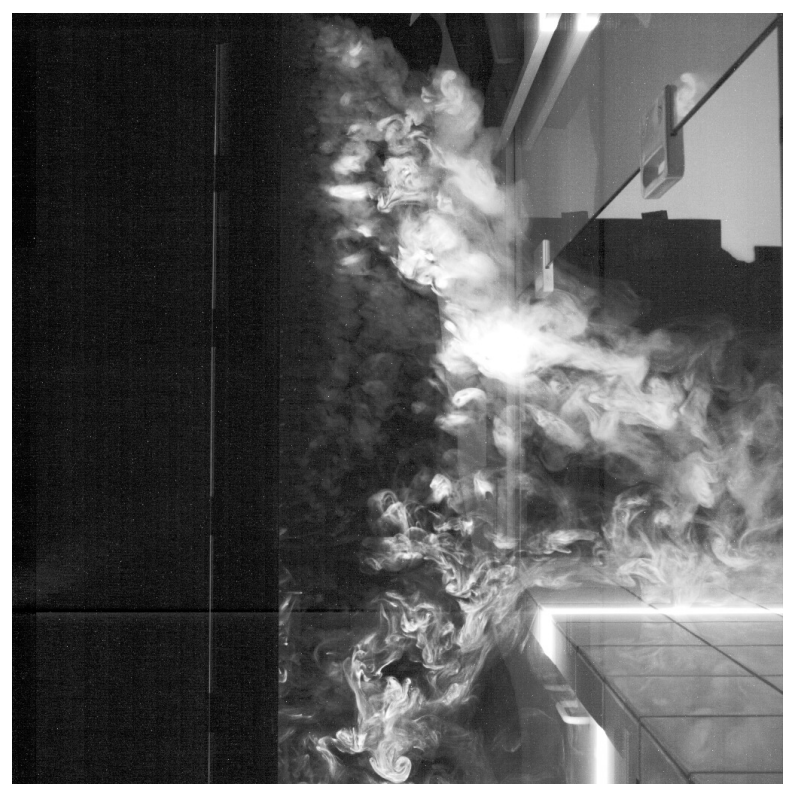

(b)

Figure 2. Instantaneous snapshots from HFSB image sequence (a) and smoke image sequence (b).

\subsection{Motion estimation from image sequences method}

As mentioned above, we seeded the region of interest with a tracer in order to make the fluid flow visible. With a wellchosen tracer that can closely follow the fluid motion, we can assume that the tracer motion equals the fluid motion. In this section we expose our strategy to estimate the fluid motion from observations of tracer displacements during an image sequence. This issue is frequently addressed, in PIV community, by a correlation based processing between two subsequent images. In practice the images are divided into several interrogation windows. For each interrogation window in the image 1 a correlation product is computed 
with the corresponding interrogation window in the image 2. The location of the correlation peak then indicates the predominant displacement of the particles contained within the interrogation window. Therefore there is only one vector per interrogation window. The resolution of the vector field can eventually be increased by using an interrogation window overlap. As mentioned in section 1 the correlation is not very performant with scalar images. Generally the correlation is to weak to be distinguished from noise. In our case, to compute the motion from two successive snapshots we chose to use an optical flow approach. Optical flow is a method that comes from the computer vision community. Its principle was firstly introduced in [Horn and Schunck (1981)]. Precisely what is called optical flow is the apparent motion between two successive frames of an image sequence. Optical flow is based on an observation model that links the spatio-temporal tracer luminance variations to the apparent motion field. The original model is based on brightness conservation assumption which supposes that a moving object keeps its luminance during motion. Mathematically it can be written:

$$
I(\boldsymbol{x}+\Delta \boldsymbol{x}, t+\Delta t)=I(\boldsymbol{x}, t)
$$

Assuming small displacements, equation (1) can be linearized and then reads:

$$
\frac{\partial I}{\partial t}+\boldsymbol{v} \nabla I=0
$$

where $\boldsymbol{v}=(u, v)^{T}$ is the two-components motion field and $I$ is the image grey levels. One of the benefits of using optical flow is that it provides a dense motion field (1 vector/pixel), much better than correlation resolution limited to 0.125 vector/pixel in the best case. Moreover, compared to correlation approaches optical flow provides a framework to use physical-based models, which improves the estimation of fluid motion. As a matter of fact the formulation of equation (2) works well for rigid objects motion but it does not model the complexity of fluid motion. An exhaustive review of optical flow advances dedicated to fluid motion estimation has been published in [Heitz et al. (2010)]. Several observation models have been investigated to cope with turbulent fluid motion. In our case we developed a largescale observation model that takes into account the effects of unresolved scales and errors, on the scale of the measured velocity. In this model introduced by [Mémin (2014)], non-observed scales are expressed thanks to stochastic formalism. Concretely the unresolved scales (i.e. subpixel scales) are modeled by a random Brownian term. From this stochastic model a new passive scalar transport equation is derived:

$$
\mathrm{d}_{t} I+\boldsymbol{v}^{\star} \mathrm{d} t \cdot \nabla I-\frac{1}{2} \nabla \cdot(\boldsymbol{a} \nabla I) \mathrm{d} t=0,
$$

where $\boldsymbol{a}$ is a $2 \times 2$ matrix representing the variance of the subgrid scale velocity. The velocity $\boldsymbol{v}^{\star}=\boldsymbol{v}-\frac{1}{2} \boldsymbol{\nabla} \cdot \boldsymbol{a}$ is a modified velocity due to inhomogeneities of the tensor $\boldsymbol{a}$. This new stochastic multi-scale data term has already shown good performances for the motion estimation of turbulent flows in [Cai et al. (2018)] and in [Schuster et al. (2018)]. Concerning the optimization strategy we chose a local resolution scheme exposed by [Lucas and Kanade (1981)]. For each pixel the corresponding vector is computed by a least-squares minimization of the right hand term of equation (3) in a close neighborhood of the pixel. This approach is well-suited for parallelization (because each pixel is processed independently), this is interesting for realtime in situ applications.

\section{Results and discussion}

\subsection{Mean flow analysis}

The figure 3 shows two mean velocity fields obtained from HFSB seeding and smoke seeding. The mean was computed from 3000 instantaneous velocity fields. The color indicates the magnitude $\|\boldsymbol{v}\|=\sqrt{u^{2}+v^{2}}$ of the velocity. As we can see, the two presented fields are not exactly the same although they are supposed to represent the same flow. In the central region of the sash opening the two mean fields are quite similar, but at the bottom and at the top they differ. Inside the fume hood on the bench the velocity is null for smoke seeding. Idem upward inside the fume hood. It comes from the fact that the smoke was not well distributed everywhere during the image acquisition process. Some areas were difficult to seed inside the fume hood. As a matter of fact the smoke generator was located outside the fume hood and was driven manually. It was not easy to constantly cover the entire zone of interest. Moreover images need to have a lot of luminance gradients, so the smoke generation should be pulsed in order to create smoke structures and not a diffuse haze. For this reasons some regions like bottom or top of the fume hood were not always well seeded leading to underestimated vectors. In opposition the HFSB generation was run automatically so it was easier to insure a dense homogeneous and continuous seeding in the whole field of observation. The figure 4 shows vertical profiles of the mean velocity magnitude taken between the fume hood workbench (height $=0 \mathrm{~mm}$ ) and the sliding glass inferior 

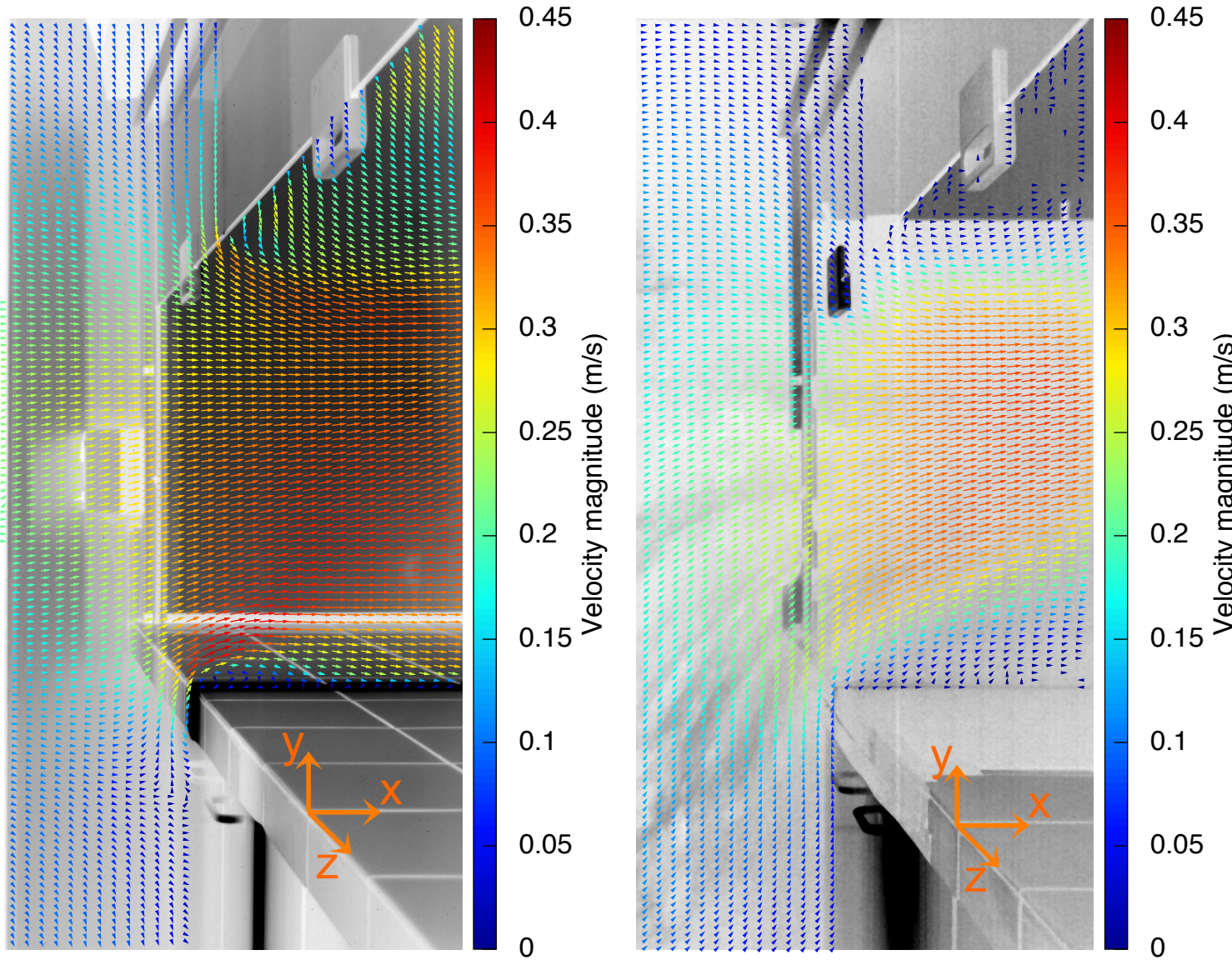

Figure 3. Mean velocity fields obtained for an opening height of $500 \mathrm{~mm}$ with a continuous light. Results for two kinds of seeding are shown here: HFSB (left) and smoke (right).

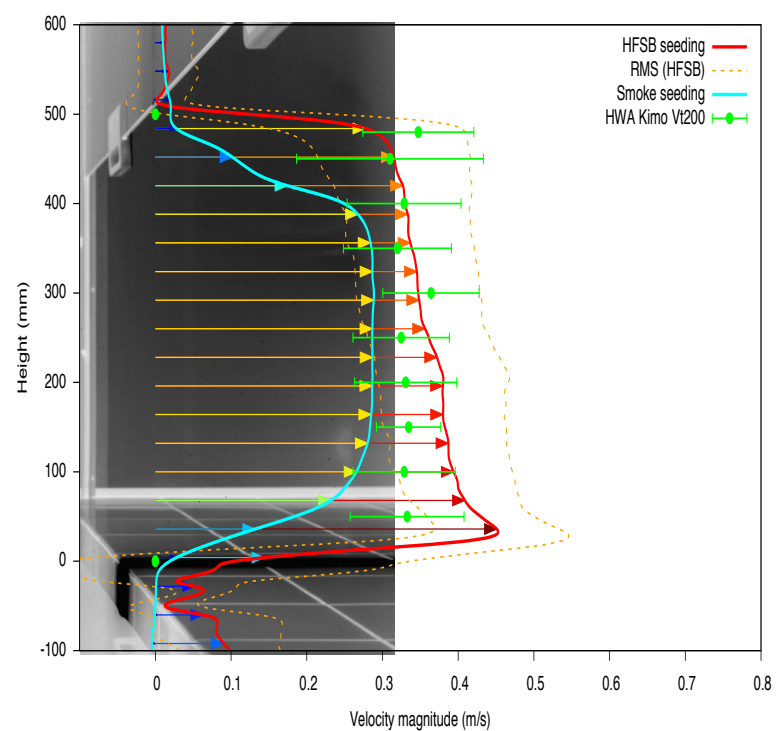

Figure 4. Vertical profiles of $\bar{U}$, the mean longitudinal velocity component taken at the glass door position on the $x$-axis.

Results from HFSB seeding (red line and orange dashed-line) and smoke seeding (blue line) are compared to sparse hot-wire anemometry measurements (green errorbars).

edge (height $=500 \mathrm{~mm}$ ). The goal is to compare our method to a typical measurement that is commonly done during periodical controls namely spot hot-wire anemometry. As we can see, the HFSB seeding gave results close enough to the HWA (except at extremities where the air speed was too weak to be measured by the hot-wire anemometer) but with a clearly better spatial resolution. As expected, the smoke gave the global trend but slightly underestimated the velocity for the reasons mentioned above. Actually large-scale velocity measurements in a mixing layer worked very well in the wind tunnel experiment of [Schuster et al. (2018)]. As it can be seen in figure 5 (a) the smoke was well-distributed and presented a lot of gradients. The mean velocity field of figure 5(b) was quite satisfying and enabled us to retrieve the main parameters of the mixing layer. Therefore, this first analysis outlines the fact that there is still a gap remaining between well conditioned experiments and on-site measurements, especially for automatically structured smoke generation.

\subsection{Turbulent flow analysis}

In the figure 6 we plot a map of the flow turbulence intensity defined as $\sqrt{\overline{{u^{\prime 2}}^{2}}} / U_{\text {out }}$ where $\overline{u^{\prime 2}}$ stands for the time average of the quadratic instantaneous fluctuations defined as $u^{\prime}(\boldsymbol{x}, t)=u(\boldsymbol{x}, t)-\overline{U(\boldsymbol{x})} . U_{\text {out }}$ stands for the global 


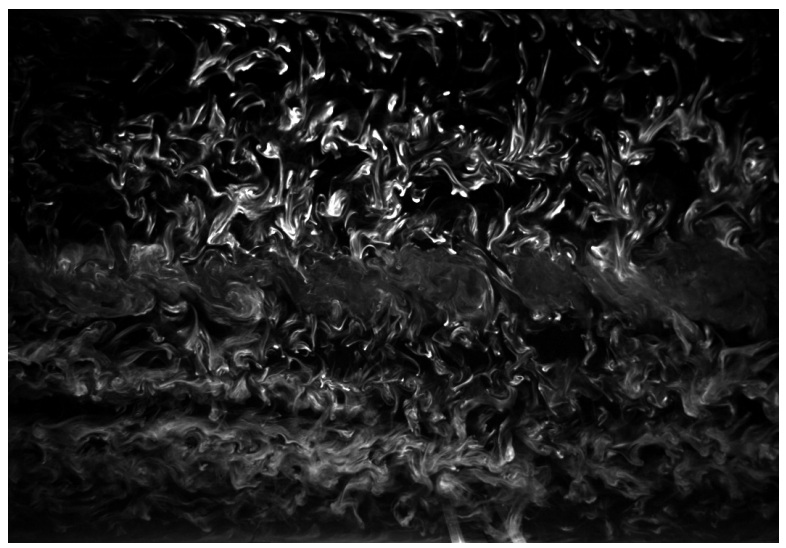

(a)

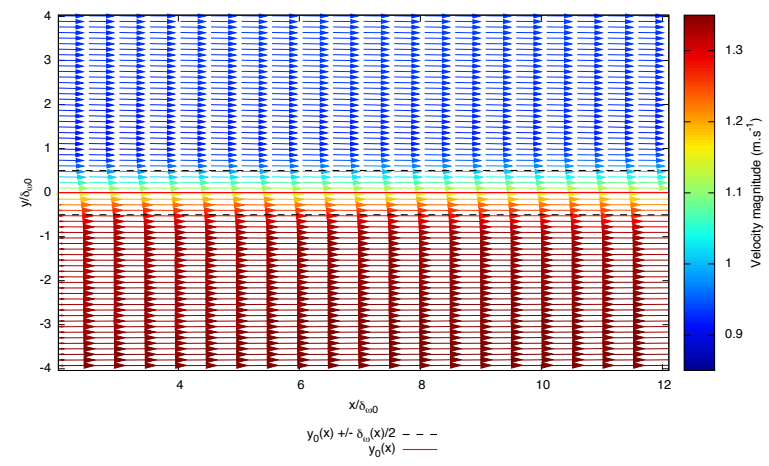

(b)

Figure 5. Snapshot of smoke seeding for a large-scale observation of a mixing layer in HABV wind tunnel of Irstea center (a). Mean velocity field of the mixing layer obtained with smoke seeding (b).

mean suction velocity (here we took $U_{\text {out }}=0.254 \mathrm{~m} / \mathrm{s}$ ). The average is computed from 3000 instantaneous velocity fields. Thanks to this representation it is easier to distinguish areas where the turbulence is stronger. In this particular case the strongest turbulent area is located just behind the edge of the bench where the flow is highly turbulent due to the bench corner wake and the bench boundary layer. Another turbulent area, located in the wake of the bottom edge of the sliding window, also presents a high level of turbulence. Finally a third area, located at the top inside the fume hood behind the glass seems to present a high level of turbulence. The turbulence activity is a sign of flow unsteadiness, it is generally linked to the presence of eddies in the flow. According to the location of these turbulent areas it can be responsible for leakages in the worst case.

So far we have just presented time averaged quantities but another big interest of an image-based method compared to sparse hot-wire anemometry is the possibility of visualizing the spatio-temporal flow evolution and thus following dynamic flow features, which are difficult to identify with HWA. The figure 7 shows an instantaneous vorticity map of

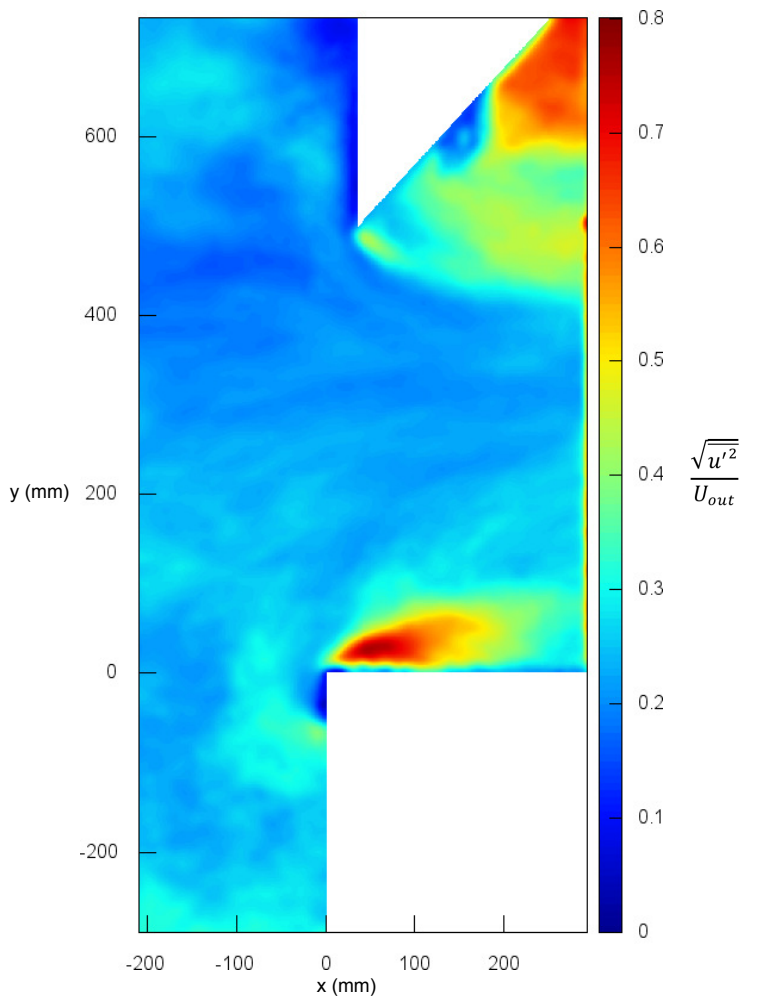

Figure 6. Reynolds stress of the $u$ component (i.e. along the $x$-axis) of the velocity.

the flow obtained from the HFSB sequence. The vorticity

$$
\omega=\frac{\partial v}{\partial x}-\frac{\partial u}{\partial y},
$$

is presented in the dimensionless form

$$
\frac{\omega H}{U_{\text {out }}},
$$

where $H=500 \mathrm{~mm}$ is the opening height and $U_{\text {out }}$ is the already mentioned global suction flow velocity. The color indicates the direction of rotation and its intensity. Strong negative vortex structures are evolving on the bench surface. In the center, the flow has a global low vorticity magnitude, however small and poorly energetic vortex structures are sparsely distributed. To our mind, these tiny vortex structures should not be confounded with spurious noise as they are advected across time and keep their coherence for most of them. Higher in the flow, strong positive vortex shedding are observable in the wake of the window edge. There is also a large recirculation formation high inside the fume hood chamber. In the figure 8 the corresponding instantaneous streamlines field is displayed at several location. A streamline is a line tangent at any point to the direction of the fluid velocity. In the global view of figure 8(a) the flow seems to be coarsely laminar in the center of the opening. When entering the enclosure, the streamlines 


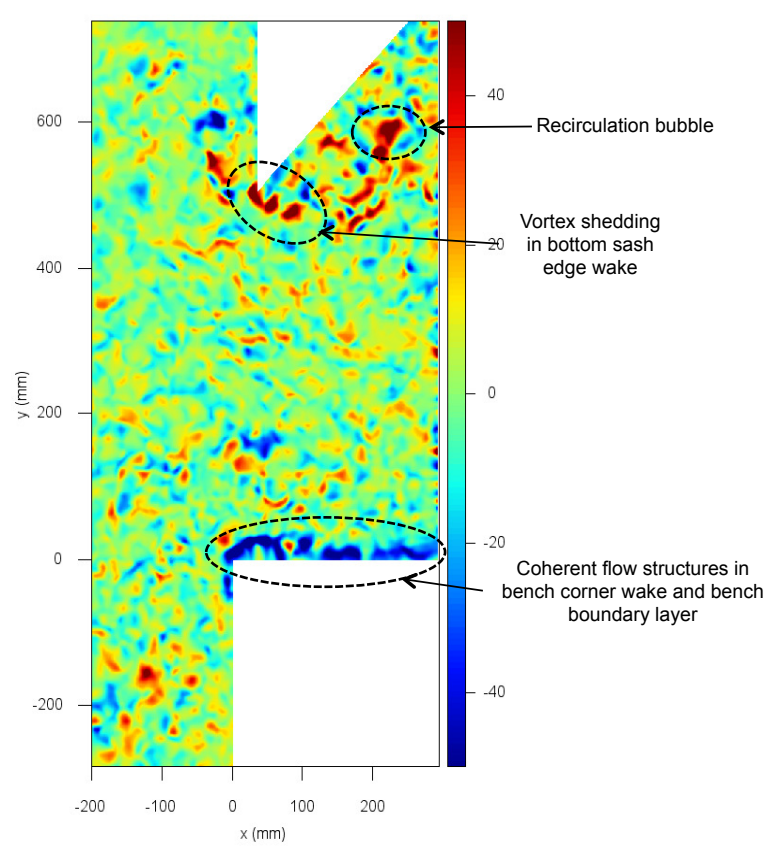

Figure 7. Instantaneous vorticity map obtained with HFSB seeding.

are getting tighter which means that the flow is accelerating. Inside the chamber the streamlines start to go upward and they deviate from each other which means that the flow is slowing down. Upward inside the chamber streamlines are curling upon themselves, a close look into this area is shown in $8(\mathrm{~b})$. Nearby obstacles or sharp edges, like in the the workbench area, streamlines also present strong curvatures $8(\mathrm{c})$. As we can see, the presented method is able to retrieve vortex structures that were already observed through smoke visualisation by [Tseng et al. (2006)] and by [Chen (2016)], like the recirculation bubble behind the rightangled bench corner or vortex shedding in the bottom edge of sash wake. The big recirculation area upward inside the chamber was also identified by [Pietrowicz et al. (2018)]. The intensity and persistence in time of such flow structures give an indication about the location of potential leakages. Without necessarily causing chemicals leaks, turbulent areas and vortex structures inside the enclosure can be responsible for loss of energy efficiency. Therefore it is crucial to detect them in order to improve fume hood design and decrease flow resistance. In our particular case no reverse flow was observed, however when looking at the HFSB image sequence we clearly saw that sometimes bubbles were trapped in the bench edge recirculation zone. Therefore there can be an accumulation of chemicals in this area. To check this, a measurement of a tracer gas concentration should be carried out to establish a correlation between recirculation areas and chemicals concentration.

\section{Conclusion}

In this study we have demonstrated the possibility of performing on-site large-scale image-based measurements on an operating laboratory fume hood. Measurements were performed on a single vertical plane perpendicular to the fume hood opening. We tested two seeding systems: HFSB and smoke. The results obtained with bubbles were better than results obtained with smoke. The smoke generator has to be improved to produce well-distributed smoke structures. Nevertheless when the smoke distribution is well structured the computed vector fields can be quite reliable as demonstrated in [Schuster et al. (2018), Schuster et al. (2019)].

Thanks to our method we were able to retrieve a timeresolved velocity field over an extended area. From the sequence of instantaneous velocity fields we then could compute the mean velocity field and compare to hot-wire anemometry measurements. The presented method offers some advantages compared to hot-wire anemometry: for a similar accuracy (bubble case only), we got a large distribution of two-components velocities over a whole plane when for HWA we only got one-component sparse spot measurements. We also could quantify the turbulence intensity and identify some instantaneous aerodynamic features of the flow like recirculation bubbles or coherent flow structures. This information about aerodynamic features and turbulence can be very useful to detect leaks, but it also brings to us a better understanding of ventilation devices and could help to improve their design in order to optimize their efficiency.

To go further in the fume hood characterisation, the next step could be to perform our method on several planes (vertical and horizontal ones) in order to have an exhaustive 3D mapping of the flow like in [Pietrowicz et al. (2018)] or in [Li et al. (2015)]. It would be also interesting to test the method in presence of a manikin standing in front the opening to see the effect of the presence of a human body on the flow. Concerning the material, the use of a low-cost camera or a smartphone camera should be investigated. In conclusion, this study let us believe that the exposed method could be widely applied for periodical control of laboratory fume hoods or other ventilation systems inside buildings. To our minds it delivers a much more complete flow characterisation than actual periodical controls based on sparse averaged hot-wire anemometry. 


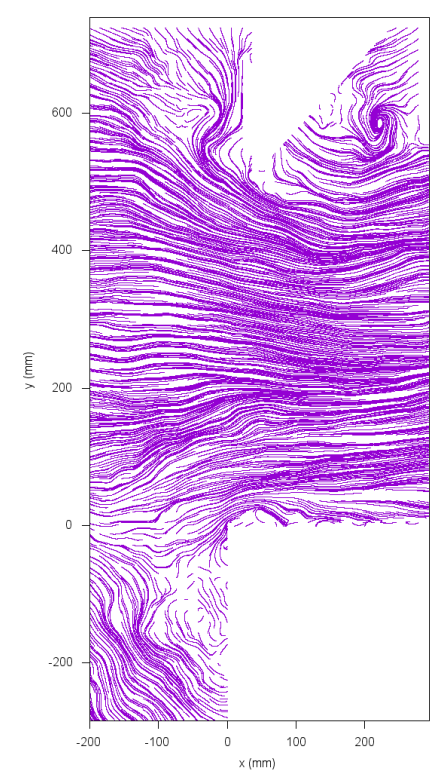

(a)

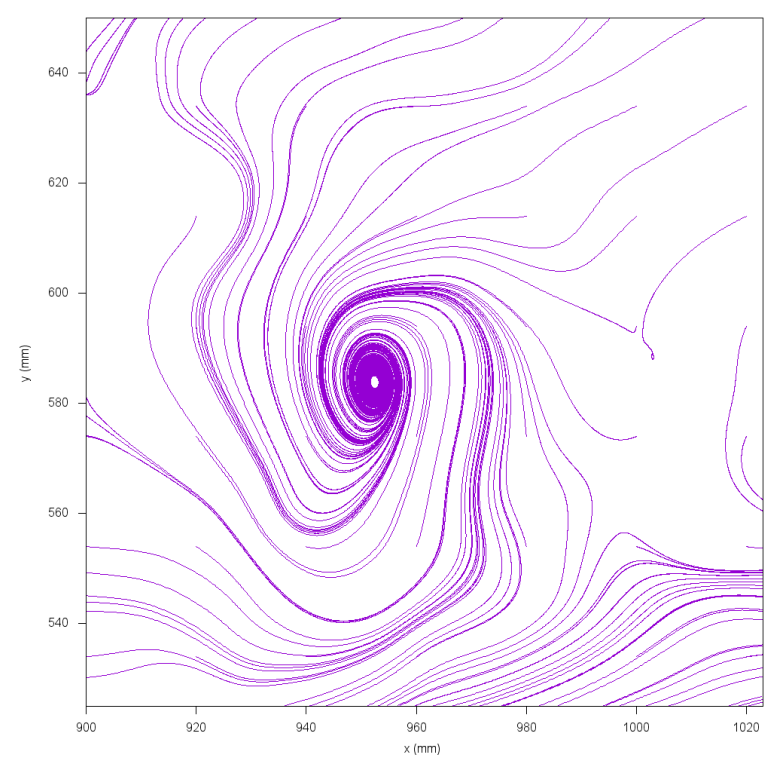

(b)

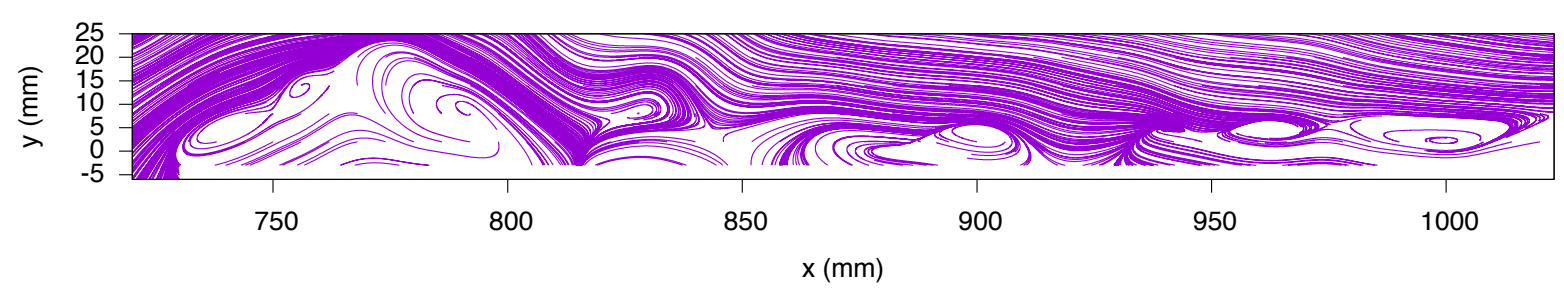

(c)

Figure 8. Instantaneous streamline field. Global view (a), zoomed view into a large recirculation area (b) and zoomed view into the bench boundary layer (c).

\section{Declarations}

\section{Authors' contributions}

All authors contributed equally in the preparation of this manuscript.

\section{Competing interests}

The authors declare that they have no competing interests.

\section{Acknowledgements}

This work was supported by ANRT, ITGA, Irstea and INRIA.

\section{References}

Adrian RJ (1991) Particle-Imaging Techniques for Experimental Fluid Mechanics. Annual Review of Fluid Mechanics : 44.

Adrian RJ and Westerweel J (2011) Particle Image Velocimetry. Cambridge University Press. ISBN 978-0521-44008-0.
Cai S, Mémin E, Dérian P and Xu C (2018) Motion estimation under location uncertainty for turbulent fluid flows. Experiments in Fluids 59(1).

Cao G, Awbi H, Yao R, Fan Y, Sirén K, Kosonen R and Zhang JJ (2014) A review of the performance of different ventilation and airflow distribution systems in buildings. Building and Environment 73: 171-186.

Chen JK (2016) Installation of a flow control device in an inclined air-curtain fume hood to control wake-induced exposure. Journal of Occupational and Environmental Hygiene 13(8): 588-597.

Chen W, Li J, Wang C, Dai X and Liu J (2018) 2D-PIV measurement of range hood-driven flow in a domestic kitchen. Energy and Buildings 177: 64-76.

Heitz D, Mémin E and Schnörr C (2010) Variational fluid flow measurements from image sequences: synopsis and perspectives. Experiments in Fluids 48(3): 369-393.

Horn BKP and Schunck BG (1981) Determining Optical Flow. Artificial Intelligence 17: 185-203. 
Jiang H, Wang Q, Zheng TF, Tu CX and Zhang K (2016) PIV measurement of internal flow field in a range hood. Energy And Mechanical Engineering - Proceedings Of 2015 International Conference 570-575.

Li J, Cao X, Liu J, Wang C and Zhang Y (2015) Global airflow field distribution in a cabin mock-up measured via large-scale 2d-PIV. Building and Environment 93: 234244.

Liu W, Liu D and Gao N (2017) CFD study on gaseous pollutant transmission characteristics under different ventilation strategies in a typical chemical laboratory. Building and Environment 126: 238-251.

Lucas BD and Kanade T (1981) An Iterative Image Registration Technique with an Application to Stereo Vision. In: Proceedings of the 7th International Joint Conference on Artificial Intelligence, volume 2. Vancouver, BC, Canada: Morgan Kaufmann Publishers Inc., pp. 674-679.

Mémin E (2014) Fluid flow dynamics under location uncertainty. Geophysical \& Astrophysical Fluid Dynamics 108(2): 119-146.

Pietrowicz S, Kolasiski P and Pomorski M (2018) Experimental and numerical flow analysis and design optimization of a fume hood using the CFD method. Chemical Engineering Research and Design 132: 627643.

Raffel M, Willert CE, Scarano F, Kähler CJ, Wereley ST and Kompenhans J (2018) Particle Image Velocimetry: A Practical Guide. 3rd edition. Heidelberg ; New York: Springer. ISBN 978-3-319-68851-0.

Scarano F, Ghaemi S, Caridi GCA, Bosbach J, Dierksheide U and Sciacchitano A (2015) On the use of helium-filled soap bubbles for large-scale tomographic PIV in wind tunnel experiments. Experiments in Fluids 56(2).

Schuster R, Heitz D and Mémin E (2018) Motion Estimation under Location Uncertainty, Application to Large-scale Characterization of a Mixing Layer. In: Proceedings of the 19th International Symposium on the Application of Laser and Imaging Techniques to Fluid Mechanics. Lisbon, Portugal, p. 20.

Schuster R, Heitz D and Mémin E (2019) Motion estimation under location uncertainty, a local LucasKanade approach. Manuscript in preparation to be submitted in Experiments in Fluids .
Tseng LC, Huang RF, Chen CC and Chang CP (2006) Correlation Between Airflow Patterns and Performance of a Laboratory Fume Hood. Journal of Occupational and Environmental Hygiene 3(12): 694-706. 